\title{
SALMONELLA PNEUMONIA
}

\author{
By \\ Khalid I. Khan, M.D., Fellow in Pulmonary Diseases \\ Bashir A. Chaudhary, M.D., Associate Professor of Medicine \\ William A. Speir, M.D., Professor of Medicine \\ Section of Pulmonary Diseases \\ Department of Medicine \\ Medical College of Georgia \\ Augusta, Georgia
}

\begin{abstract}
Pulmonary Salmonella infections are rarely encountered in the United States these days. We describe a patient who had a left lower lobe pneumonia. Salmonella typhimurium was cultured from sputum and bronchial brushings from the lesion, and agglutinins for Salmonella were positive. The patient responded well to antibiotic therapy.
\end{abstract}

\section{SALMONELLA PNEUMONIA}

Salmonella species are common pathogens in the gastrointestinal tract. The usual syndromes due to salmonella infections include gastroenteritis, typhoid fever, enteric fever, bacteremia, and localized infections, such as nephritis, and meningitis. Lung involvement in the form of bronchopneumonia, lung abscess, and empyema do occur but are rare. We describe a case of pneumonia caused by Salmonella typhirmurium and briefly review the pertinent literature.

\section{Case Presentation:}

A 58 year old tobacco farmer was admitted to the Eugene Talmadge Memorial Hospital/Medical College of Georgia for evaluation of chest pain and left lower lobe infiltrate on the chest roentgenogram. The patient had been in good health until two weeks prior to his hospitalization when he developed substernal chest pain and mild dizziness. The chest pain improved after 30 mintues but recurred three days later in the same area. The pain was worse when the patient bent forward but was not pleuritic. Three days after the onset of his initial symptoms, the patient began having shortness of breath and blood streaked sputum. The patient did not have nausea, vomiting, diarrhea, fever, night sweats, or weight loss. The patient occasionally smoked cigarettes.

Examination showed a well-developed black male in no acute distress. The vital signs were: blood Correspondence and Reprint requests:

Basir A. Chaudhary, M.D.

Associate Professor of Medicine

Section of Pulmonary Diseases Department of Medicine

Medical College of Georgia

Augusta, Georgia 30912
DOI: http://dx.doi.org/10.5915/15-4-12423

pressure $160 / 100 \mathrm{mmHg}$, pulse $80 / \mathrm{min}$, respiratory rate $18 / \mathrm{min}$, temp $37^{\circ} \mathrm{C}$. The remainder of the examination was remarkable only for bilateral basilar crackles and expiratory wheezes over the left chest. Chest roentgenogram showed emphysematous changes and a round left lower lobe infiltrate. (Figure 1A-B)

The white blood count was $14,000 / \mathrm{mm}^{3}$ with $62 \%$ polymorphonuclear cells, $37 \%$ lymphocytes, and $1 \%$ monoctyes. Urinalysis showed numerous white blood cells and numerous bacteria which were later identified as E. coli on cultures. Other routine laboratory tests were unremarkable.

The patient was unable to cough up sputum; but after instituting chest percussion and postural drainage, he produced a small amount of sputum. A gram stain of the sputum showed a few polymorphonuclear cells but no organisms; the sputum culture grew Salmonella typhimurium and $\mathrm{H}$. Parainfluenzae. A PPD skin test was negative. To rule out the possibility of a lung tumor and to obtain material for culture, the patient was bronchoscoped. No endobronchial lesions were seen and transbronchial biopsies were negative for malignancy. Bronchial secretions were obtained from the lesion under fluoroscopic control by a double lumen catheter system and grew Salmonella typhirmurium which was sensitive to ampicillin. The patient was started on ampicillin $500 \mathrm{mg}$ four times a day and was discharged from the hospital. The patient was seen in clinic five weeks after discharge. He was completely asymptomatic, and a chest roentgenogram showed marked clearing of the infiltrate. Salmonella agglutinins drawn in clinic showed a salmonella $\mathrm{H}$ titer of $1: 80$ and salmonella 0 titer of $1: 40$.

\section{DISCUSSION}

The most common Salmonella serotypes isolated in the United States in 1978, as reported by the Centers for Disease Control, were S. typhimurium S. heidelberg, and S. enteritidis. (1) Salmonella infections present in four different clinical forms: 1) gastroenteritis; 2) typhoidal or septic syndrome; 3 ) 


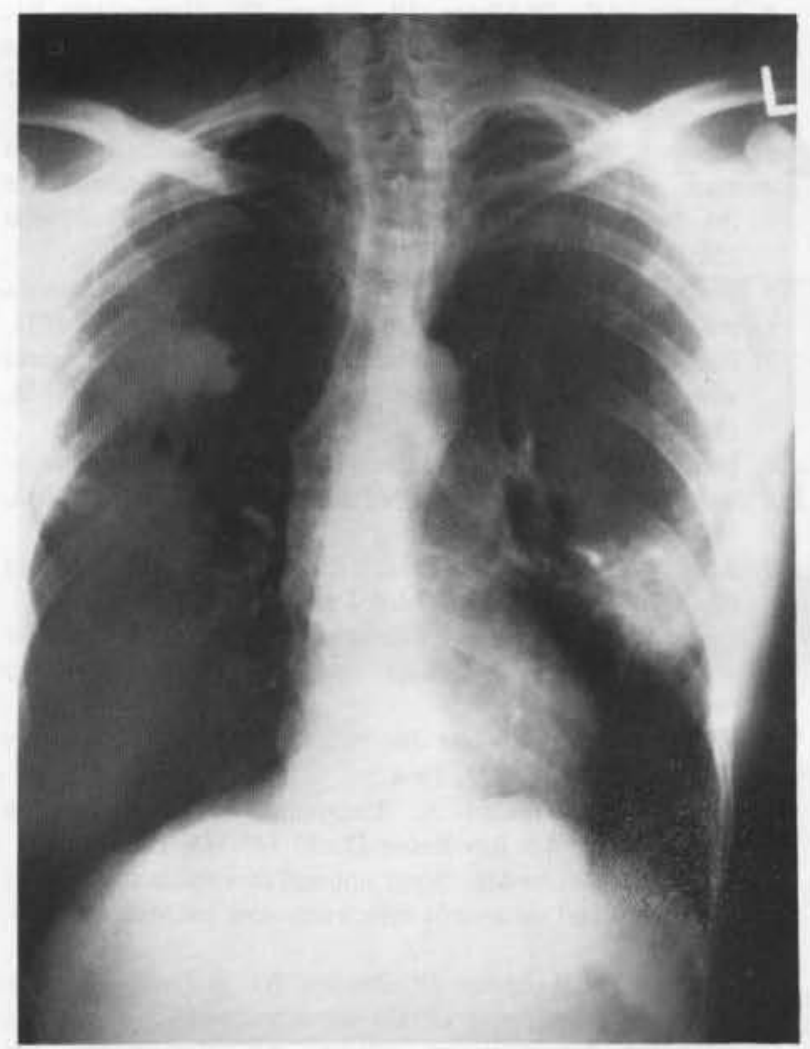

focal infections; and 4) chronic carrier state.

The incidence of focal pulmonary infection is very low. Occasional case reports of salmonella pleuropulmonary infections have been described. (2-4) In a review of 7,779 cases of salmonella infections, 5,312 $(68.3 \%)$ patients had gastroenteritis, 686 patients $(8.8 \%)$ had typhoidal or septic syndrome, and $572(7.4 \%)$ patients had focal infections. Only 84 infections ( $1 \%$ of total salmonella infections) involved the respiratory system ( $14.8 \%$ of focal infections). (5) Many cases of salmonella tonsillitis and otitis media were included in the category of focal respiratory infections, however. The most common organisms which involved the lung were $\mathrm{S}$. cholerasuis, S. typhimurium and S. paratyphi C.

The majority of patients who develop Salmonella pneumonia are over the age of fifty. Despite the fact that Salmonella is predominantly a gastrointestinal tract pathogen, more than half of the patients with Salmonella pneumonia do not have abdominal complaints and their illness is not preceded by gastroenteritis. (6) The incidence of Salmonella infection is increased in patients with malignancy, especially those with malignant lymphomas and leukemias. $(7,8,9)$

Pulmonary involvement due to salmonella is usually manifest as a bronchopneumonia; however, there

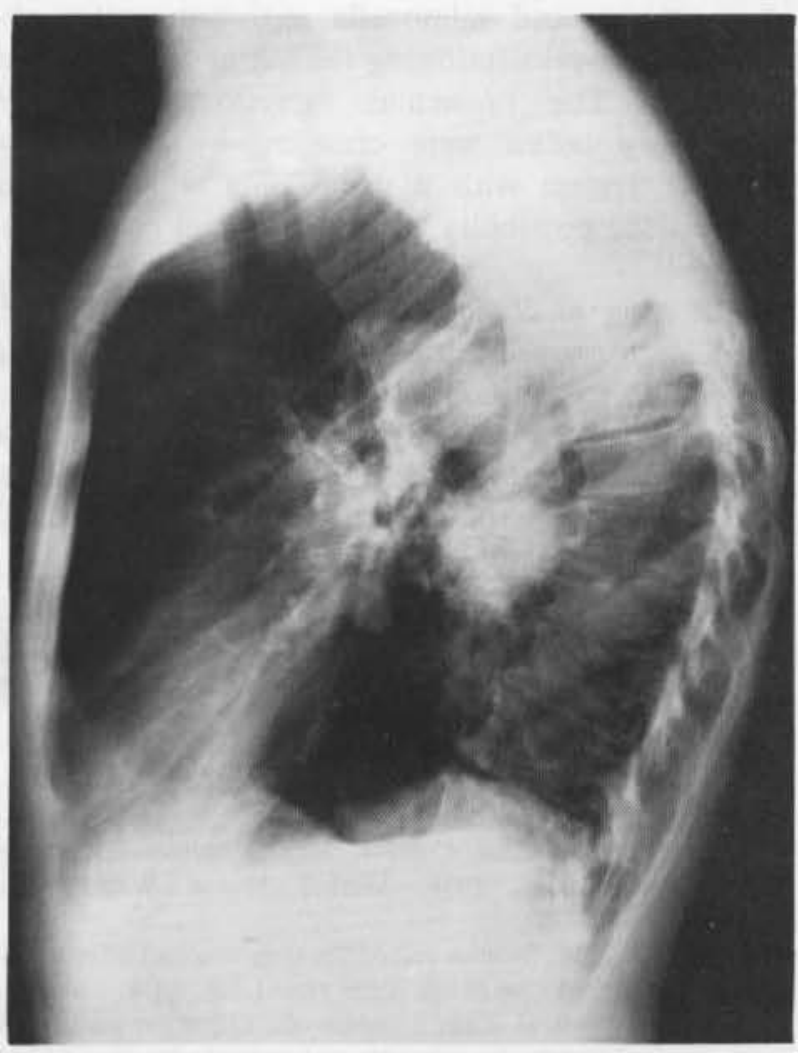

are no characteristic roentgenographic features. Rarely a miliary pattern on chest roentgenogram may be seen with salmonella bacteremia. (10) Most patients with Salmonella pneumonia are febrile; however, leukocytosis may be absent. Other thoracic manifestations of salmonella infection include lung abscess, empyema, bronchopleural fistula and costochondritis. (11-13)

All Salmonella have at least two antigens; the somatic 0 and flagella $\mathrm{H}$, both of which can induce the formation of serum agglutinins. Antibodies against somatic antigens appear early in the disease and disappear earlier than those of the flagella antigens. The titers vary at different stages of the disease and in different patients; hence it is difficult to state a certain diagnostic serum titer. It is generally agreed that a four-fold rise or fall is more reliable than a single determination. In the presence of appropriate history and clinical findings a titer of 1:80 is considered suggestive and a titer of $1: 160$ or more is diagnostic. (6) The above does not hold true if the patient has been recently immunized or has a history of recent infection.

The diagnosis of salmonella pulmonary infection in our patient was well established. Salmonella was cultured from the expectorated sputum and bronchial brush specimen obtained from the lesion under 
fluoroscopy, and salmonella agglutinins titer obtained five weeks following the initial diagnosis was elevated. The bronchial secretions from the pulmonary lesion were obtained by the double catheter system with a distal plug which greatly reduces the possibility of oral bacterial contamination (14).

The drug of choice in treating salmonella infections is chloramphenical, beginning with a loading dose of 15 to $20 \mathrm{mg} / \mathrm{kg}$ of body weight followed by 50 to $60 \mathrm{mg} / \mathrm{kg}$ of body weight $/ 24$ hours in four divided doses. Salmonella sensitive to ampicillin can be treated with ampicillin, the dosage being 60 to 80 $\mathrm{mg} / \mathrm{kg}$ body/weight/day.

\section{ACKNOWLEDGEMENTS}

The authors wish to thank Carol Purks for her secretarial assistance.

\section{References}

1. Centers for Diesase Control. Human salmonella isolates - United States, 1978. Morbid Mortal Weekly Rep 28:618-620, 1980.

2. Bullowa JGM: Bacillus suipestifer (hog cholera) infection of the lung. Med Clin North Amer 12:691-694, 1928.

3. Cohen L, Fink H, Gray I: Salmonella suipestifer bacteremia with pericarditis, pneumonitis and pleural effusion. JAMA 107:331-333, 1936.
4. Ingegno AP, D'Albora JB, Edson JN, Granquinto PJ: Pneumonia associated with acute salmonellosis; report of a case of salmonella bronchopneumonia and fourteen cases of interstitial pneumonia. Arch Int Med 81:476-486, 1948.

5. Saphra I, Winter J: Clinical manifestations of salmonellosis in man. An evaluation of 7,779 human infections identified at the New York Salmonella Center. New Engl J of Med 256:1128-1134, 1957.

6. Weiss W, Wisenberg G, Harrison F: Salmonella pleuropulmonary disease. Amer J Med Sci 233:487-496, 1957.

7. Berkeley D, Mangels J: Salmonella pneumonia in a patient with carcinoma of the lung. Am J Clin Patho 74:476-478, 1980.

8. Han T, Sokal J, Neter E; Salmonellosis in disseminated malignant diseases. (1959-1965). N Engl J of Med 276:1045-1052, 1967.

9. Abeloff M, Densen P, Lenhard R: Salmonella empyema and Hodgkin's disease. South Med J 68:363-366, 1975.

10. Greenspan RH, Feinberg SB: Salmonella bacteremia: A case with miliary lung lesions and spondylitis. Radiology 68:860-862, 1957.

11. Hahne O: Lung abscess due to salmonella typhi. Am Rev Respir Dis 89: 566-571, 1964.

12. Kuncaitis J, Okutan A: Empyema due to salmonella typhimurium. Am Rev Respir Dis 83:741-743, 1961.

13. Minor GR, White ML: Some unusual thoracic complications of typhoid and salmonella infections. Ann Int Med 24:27-39, 1946.

14. Wimberly N, Leendert JF, Bartlett JG: A fiberoptic bronchoscopy technique to obtain uncontaminated lower airway secretions for bacterial culture. Am Rev Respir Dis 119:337-343, 1979. 